\title{
$\underline{\text { Vector Potential for a Single Helical Current Conductor }}$
}

\author{
T. Tominaka \\ RIKEN
}

(The Institute of Physical and Chemical Research)

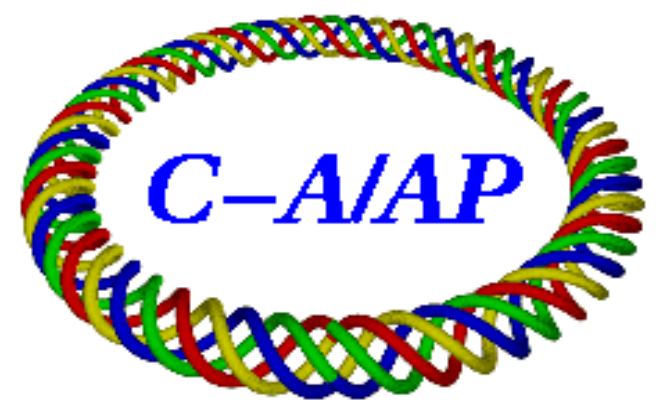

Collider-Accelerator Department Brookhaven National Laboratory Upton, NY 11973 


\title{
Vector Potential for a Single Helical Current Conductor
}

\author{
T. Tominaka* \\ RIKEN (The Institute of Physical and Chemical Research), \\ 2-1, Hirosawa, Wako, Saitama, 351-0198, Japan
}

\section{Abstract}

The expressions for vector potential of a single helical conductor have been derived from the definitive integral form, treating a single helical current as a current distribution on the surface of a infinitely thin cylinder due to the delta function.

Keywords : Helical coil; Vector potential

\section{INTRODUCTION}

There are a plenty of helical conductors or coils in various fields of the electrical instruments. The analytical expression for the divergence-free vector potential of a single helical conductor is useful for the evaluation of the magnetic energy and the magnetic flux enclosed by the helical conductor. Following the Caspi's mathematical treatment for the multipole field due to the helical wiggler magnet [1], the expressions for vector potential due to a single helical current carrying thin conductor can be directly derived from the definitive integral form.

\section{EXPRESSIONS FOR VECTOR POTENTIAL}

\subsection{Analytical expression of vector potential for a helical conductor}

The vector potential can be directly derived from the following integral form of the definition.

$$
\vec{A}=\frac{\mu_{0}}{4 \pi} \int \frac{\vec{j} d S^{\prime}}{\left|\vec{r}-\overrightarrow{r^{\prime}}\right|}
$$

\footnotetext{
${ }^{*}$ Corresponding author. Tel.: +81-48-467-9463; Fax: +81-48-461-5301. E-mail address: tominaka@postman.riken.jp (T. Tominaka).
} 
where a single helical current located at $(r=a, \theta=\varphi, z=0)$ with a pitch length $L(=2 \pi / k)$, as shown in Fig. 1, can be treated as a current distribution on the surface of a infinitely thin cylinder due to the delta function as follows,

$$
\begin{aligned}
& j_{z}=\frac{I}{a} \delta\left(\theta^{\prime}-\varphi-k z^{\prime}\right)=\frac{I}{2 \pi a}\left\{1+2 \sum_{n=1}^{\infty} \cos \left[n\left(\theta^{\prime}-\varphi-k z^{\prime}\right)\right]\right\} \\
& j_{\theta}=\frac{I}{a} k a \delta\left(\theta^{\prime}-\varphi-k z^{\prime}\right)=\frac{I k}{2 \pi}\left\{1+2 \sum_{n=1}^{\infty} \cos \left[n\left(\theta^{\prime}-\varphi-k z^{\prime}\right)\right]\right\}
\end{aligned}
$$

Furthermore, the distance between a field point $(\mathrm{r}, \theta, \mathrm{z})$ and a source point (a, $\left.\theta^{\prime}, \mathrm{z}^{\prime}\right)$ of a current distribution related to a single helical current, can be expressed as follows,

$$
\left|\vec{r}-\overrightarrow{r^{\prime}}\right|=\sqrt{a^{2}+r^{2}+\left(z-z^{\prime}\right)^{2}-2 a r \cos \left(\theta-\theta^{\prime}\right)}
$$

In addition, the relation between the unit vectors on the circular cylindrical system of both a field point and a source point as shown in Fig. 2, can be expressed as follows,

$\hat{z}^{\prime}=\hat{z}$

$\hat{\theta}^{\prime}=\sin \left(\theta-\theta^{\prime}\right) \hat{r}+\cos \left(\theta-\theta^{\prime}\right) \hat{\theta}$

\subsection{The $z$ component of the vector potential}

The $\mathrm{z}$ component of the vector potential can be expressed with the following integral form,

$$
\begin{aligned}
& A_{z}(r, \theta, z)=\frac{\mu_{0}}{4 \pi} \iint \frac{I}{2 \pi a}\left\{1+2 \sum_{n=1}^{\infty} \cos \left[n\left(\theta^{\prime}-\varphi-k z^{\prime}\right)\right]\right\} \\
& \times \frac{a d \theta^{\prime} d z^{\prime}}{\sqrt{a^{2}+r^{2}+\left(z-z^{\prime}\right)^{2}-2 \operatorname{arcos}\left(\theta-\theta^{\prime}\right)}}
\end{aligned}
$$

With the introduction of the following working variables,

$$
\begin{aligned}
& z-z^{\prime}=s \\
& \theta-\theta^{\prime}=t
\end{aligned}
$$

the term of the integrand can be reduced to the following from, 
$\cos \left[n\left(\theta^{\prime}-\varphi-k z^{\prime}\right)\right]=$

$\cos [n(\theta-\varphi-k z)] \cos [n(t-k s)]$

$+\sin [n(\theta-\varphi-k z)] \sin [n(t-k s)]$

$=\cos [n(\theta-\varphi-k z)]\{\cos (n t) \cos (n k s)+\sin (n t) \sin (n k s)\}$

$+\sin [n(\theta-\varphi-k z)]\{\sin (n t) \cos (n k s)-\cos (n t) \sin (n k s)\}$

Neglecting the terms those result zero, the $\mathrm{z}$ component of the vector potential can be reduced to the following from.

$$
\begin{aligned}
& A_{z}(r, \theta, z)=\frac{\mu_{0} I}{8 \pi^{2}} \int_{0}^{2 \pi} \int_{-\infty}^{\infty} \\
& \times\left\{1+2 \sum_{n=1}^{\infty} \cos [n(\theta-\varphi-k z)] \cos (n t) \cos (n k s)\right\} \\
& \times \frac{d t d s}{\sqrt{a^{2}+r^{2}+s^{2}-2 a r \cos t}}
\end{aligned}
$$

Furthermore, from the mathematical manipulations, the following result of the integration can be obtained [2,3].

$$
\begin{aligned}
& \int_{0}^{2 \pi} \int_{-\infty}^{\infty} \frac{d t d s}{\sqrt{a^{2}+r^{2}+s^{2}-2 a r \cos t}} \\
& =\int_{0}^{2 \pi}\left\{2 \ln \sqrt{a^{2}+r^{2}-2 \operatorname{arcos} t}+C\right\} d t \\
& =\left\{\begin{array}{l}
-2 \int_{0}^{2 \pi}\left\{\ln a-\sum_{n=1}^{\infty} \frac{1}{n}\left(\frac{r}{a}\right)^{n} \cos n t\right\} d t, \quad r \leq a \\
-2 \int_{0}^{2 \pi}\left\{\ln r-\sum_{n=1}^{\infty} \frac{1}{n}\left(\frac{a}{r}\right)^{n} \cos n t\right\} d t, \quad r \geq a
\end{array}\right. \\
& = \begin{cases}-4 \pi \ln a, & r \leq a \\
-4 \pi \ln r, & r \geq a\end{cases}
\end{aligned}
$$

Similarly, the following result of the integration can be obtained $[4,5]$. 


$$
\begin{aligned}
& \left.\int_{0}^{2 \pi} \int_{-\infty}^{\infty} \frac{\cos (n t) \cos (n k s) d t d s}{\sqrt{a^{2}+r^{2}+s^{2}-2 a r \cos t}}\right) \cos (n t) d t \\
& =2 \int_{0}^{2 \pi} K_{0}\left(n k \sqrt{a^{2}+r^{2}-2 a r \cos t}\right) \\
& = \begin{cases}4 \int_{0}^{2 \pi} K_{n}(n k a) I_{n}(n k r) \cos (n t) \cos (n t) d t, & r \leq a \\
4 \int_{0}^{2 \pi} I_{n}(n k a) K_{n}(n k r) \cos (n t) \cos (n t) d t, & r \geq a\end{cases} \\
& = \begin{cases}4 \pi K_{n}(n k a) I_{n}(n k r), & r \leq a \\
4 \pi I_{n}(n k a) K_{n}(n k r), & r \geq a\end{cases}
\end{aligned}
$$

Finally, the following expressions for the $\mathrm{z}$ component of the vector potential are obtained in the form of the infinite sum,

for $\mathrm{r} \leq \mathrm{a}$,

$$
A_{z}(r, \theta, z)=-\frac{\mu_{0} I}{2 \pi} \ln a+\frac{\mu_{0} I}{\pi} \sum_{n=1}^{\infty} K_{n}(n k a) I_{n}(n k r) \cos [n(\theta-\varphi-k z)]
$$

for $\mathrm{r} \geq \mathrm{a}$,

$A_{z}(r, \theta, z)=-\frac{\mu_{0} I}{2 \pi} \ln r+\frac{\mu_{0} I}{\pi} \sum_{n=1}^{\infty} I_{n}(n k a) K_{n}(n k r) \cos [n(\theta-\varphi-k z)]$

\subsection{The r component of the vector potential}

The $\mathrm{r}$ component of the vector potential can be expressed with the following integral form,

$$
\begin{aligned}
& A_{r}(r, \theta, z)=\frac{\mu_{0}}{4 \pi} \iint \frac{I k}{2 \pi}\left\{1+2 \sum_{n=1}^{\infty} \cos \left[n\left(\theta^{\prime}-\varphi-k z^{\prime}\right)\right]\right\} \\
& \times \frac{\sin \left(\theta-\theta^{\prime}\right) a d \theta^{\prime} d z^{\prime}}{\sqrt{a^{2}+r^{2}+\left(z-z^{\prime}\right)^{2}-2 \operatorname{arcos}\left(\theta-\theta^{\prime}\right)}}
\end{aligned}
$$

Neglecting the terms those result zero, the $r$ component of the vector potential can be reduced to the following from. 


$$
\begin{aligned}
& A_{r}(r, \theta, z)=\frac{\mu_{0} I}{8 \pi^{2}} k a \int_{0}^{2 \pi} \int_{-\infty}^{\infty} \\
& \times\left\{1+2 \sum_{n=1}^{\infty} \sin [n(\theta-\varphi-k z)] \sin (n t) \cos (n k s)\right\} \\
& \times \frac{\sin t d t d s}{\sqrt{a^{2}+r^{2}+s^{2}-2 a r \cos t}}
\end{aligned}
$$

Furthermore, from the mathematical manipulations, the following results of the integration can be obtained [2-5].

$$
\begin{aligned}
& \int_{0}^{2 \pi} \int_{-\infty}^{\infty} \frac{\sin t d t d s}{\sqrt{a^{2}+r^{2}+s^{2}-2 a r \cos t}} \\
& =\int_{0}^{2 \pi}\left\{-2 \ln \sqrt{a^{2}+r^{2}-2 a r \cos t}+C\right\} \sin t d t \\
& = \begin{cases}-2 \int_{0}^{2 \pi}\left\{\ln a-\sum_{n=1}^{\infty} \frac{1}{n}\left(\frac{r}{a}\right)^{n} \cos n t\right\} \sin t d t, & r \leq a \\
-2 \int_{0}^{2 \pi}\left\{\ln r-\sum_{n=1}^{\infty} \frac{1}{n}\left(\frac{a}{r}\right)^{n} \cos n t\right\} \sin t d t, & r \geq a\end{cases} \\
& =0 \\
& \int_{0}^{2 \pi} \int_{-\infty}^{\infty} \frac{\sin (n t) \cos (n k s) \sin t d t d s}{\sqrt{a^{2}+r^{2}+s^{2}-2 a r \cos t}} \\
& =2 \int_{0}^{2 \pi} K_{0}\left(n k \sqrt{a^{2}+r^{2}-2 a r \cos t}\right) \sin (n t) \sin t d t \\
& =\left\{2 \int_{0}^{2 \pi} \sum_{m=-\infty}^{\infty} K_{m}(n k a) I_{m}(n k r) \cos (m t)\left\{-\frac{\cos (n+1) t-\cos (n-1) t}{2}\right\} d t, \quad r \leq a\right. \\
& \left\{2 \int_{0}^{2 \pi} \sum_{m=-\infty}^{\infty} I_{m}(n k a) K_{m}(n k r) \cos (m t)\left\{-\frac{\cos (n+1) t-\cos (n-1) t}{2}\right\} d t, \quad r \geq a\right. \\
& = \begin{cases}-2 \pi\left\{K_{n+1}(n k a) I_{n+1}(n k r)-K_{n-1}(n k a) I_{n-1}(n k r)\right\} & r \leq a \\
-2 \pi\left\{I_{n+1}(n k a) K_{n+1}(n k r)-I_{n-1}(n k a) K_{n-1}(n k r)\right\} & r \geq a\end{cases}
\end{aligned}
$$

Finally, the following expressions for the $r$ component of the vector potential are obtained,

for $\mathrm{r} \leq \mathrm{a}$,

$$
\begin{aligned}
& A_{r}(r, \theta, z)=-\frac{\mu_{0} I}{2 \pi} k a \sum_{n=1}^{\infty}\left\{K_{n+1}(n k a) I_{n+1}(n k r)-K_{n-1}(n k a) I_{n-1}(n k r)\right\} \\
& \times \sin [n(\theta-\varphi-k z)]
\end{aligned}
$$

for $\mathrm{r} \geq \mathrm{a}$, 
$A_{r}(r, \theta, z)=-\frac{\mu_{0} I}{2 \pi} k a \sum_{n=1}^{\infty}\left\{I_{n+1}(n k a) K_{n+1}(n k r)-I_{n-1}(n k a) K_{n-1}(n k r)\right\}$

$\times \sin [n(\theta-\varphi-k z)]$

\subsection{The $\theta$ component of the vector potential}

The $\theta$ component of the vector potential can be expressed with the following integral form,

$$
\begin{aligned}
& A_{\theta}(r, \theta, z)=\frac{\mu_{0}}{4 \pi} \iint \frac{I k}{2 \pi}\left\{1+2 \sum_{n=1}^{\infty} \cos \left[n\left(\theta^{\prime}-\varphi-k z^{\prime}\right)\right]\right\} \\
& \times \frac{\cos \left(\theta-\theta^{\prime}\right) a d \theta^{\prime} d z^{\prime}}{\sqrt{a^{2}+r^{2}+\left(z-z^{\prime}\right)^{2}-2 \operatorname{arcos}\left(\theta-\theta^{\prime}\right)}}
\end{aligned}
$$

Neglecting the terms those result zero, the $\theta$ component of the vector potential can be reduced to the following from.

$$
\begin{aligned}
& A_{\theta}(r, \theta, z)=\frac{\mu_{0} I}{8 \pi^{2}} k a \int_{0}^{2 \pi} \int_{-\infty}^{\infty} \\
& \times\left\{1+2 \sum_{n=1}^{\infty} \cos [n(\theta-\varphi-k z)] \cos (n t) \cos (n k s)\right\} \\
& \times \frac{\cos t d t d s}{\sqrt{a^{2}+r^{2}+s^{2}-2 a r \cos t}}
\end{aligned}
$$

Furthermore, from the mathematical manipulations, the following results of the integration can be obtained [2-5].

$$
\begin{aligned}
& \int_{0}^{2 \pi} \int_{-\infty}^{\infty} \frac{\cos t d t d s}{\sqrt{a^{2}+r^{2}+s^{2}-2 a r \cos t}} \\
& =\int_{0}^{2 \pi}\left\{-2 \ln \sqrt{a^{2}+r^{2}-2 a r \cos t}+C\right\} \cos t d t \\
& =\left\{\begin{array}{l}
-2 \int_{0}^{2 \pi}\left\{\ln a-\sum_{n=1}^{\infty} \frac{1}{n}\left(\frac{r}{a}\right)^{n} \cos n t\right\} \cos t d t, \quad r \leq a \\
-2 \int_{0}^{2 \pi}\left\{\ln r-\sum_{n=1}^{\infty} \frac{1}{n}\left(\frac{a}{r}\right)^{n} \cos n t\right\} \cos n t d t, \quad r \geq a
\end{array}\right. \\
& =\left\{\begin{array}{l}
2 \pi \frac{r}{a}, \quad r \leq a \\
2 \pi \frac{a}{r}, \quad r \geq a
\end{array}\right.
\end{aligned}
$$




$$
\begin{aligned}
& \int_{0}^{2 \pi} \int_{-\infty}^{\infty} \frac{\cos (n t) \cos (n k s) \cos t d t d s}{\sqrt{a^{2}+r^{2}+s^{2}-2 a r \cos t}} \\
& =2 \int_{0}^{2 \pi} K_{0}\left(n k \sqrt{a^{2}+r^{2}-2 a r \cos t}\right) \cos (n t) \cos t d t \\
& =\left\{\begin{array}{ll}
2 \int_{0}^{2 \pi} \sum_{m=-\infty}^{\infty} K_{m}(n k a) I_{m}(n k r) \cos (m t)\left\{\frac{\cos (n+1) t+\cos (n-1) t}{2}\right\} d t, & r \leq a
\end{array}\right\} d t, \quad r \geq a \\
& 2 \pi \int_{0}^{2 \pi} \sum_{m=-\infty}^{\infty} I_{m}(n k a) K_{m}(n k r) \cos (m t)\left\{\frac{\cos (n+1) t+\cos (n-1) t}{2}\right\} \\
& = \begin{cases}2 \pi\left\{K_{n+1}(n k a) I_{n+1}(n k r)+K_{n-1}(n k a) I_{n-1}(n k r)\right\} & r \leq a \\
2 \pi\left\{I_{n+1}(n k a) K_{n+1}(n k r)+I_{n-1}(n k a) K_{n-1}(n k r)\right\} & r \geq a\end{cases}
\end{aligned}
$$

Finally, the following expressions for the $\mathrm{z}$ component of the vector potential are obtained,

for $\mathrm{r} \leq \mathrm{a}$,

$$
\begin{aligned}
& A_{\theta}(r, \theta, z)=\frac{\mu_{0} I}{4 \pi} k r+\frac{\mu_{0} I}{2 \pi} k a \sum_{n=1}^{\infty}\left\{K_{n+1}(n k a) I_{n+1}(n k r)+K_{n-1}(n k a) I_{n-1}(n k r)\right\} \\
& \times \cos [n(\theta-\varphi-k z)]
\end{aligned}
$$

for $r \geq a$,

$$
\begin{aligned}
& A_{\theta}(r, \theta, z)=\frac{\mu_{0} I}{4 \pi} k \frac{a^{2}}{r}+\frac{\mu_{0} I}{2 \pi} k a \sum_{n=1}^{\infty}\left\{I_{n+1}(n k a) K_{n+1}(n k r)+I_{n-1}(n k a) K_{n-1}(n k r)\right\} \\
& \times \cos [n(\theta-\varphi-k z)]
\end{aligned}
$$

\section{FIELD OF A SINGLE HELICAL CURRENT CONDUCTOR}

The magnetic fields $\mathrm{B}$ due to a single helical current carrying thin conductor can be obtained from the vector potential as follows,

$\vec{B}=\nabla \times \vec{A}$

As a result, following the recurrence relations of modified Bessel functions [6], the following expressions are obtained, for $\mathrm{r}<\mathrm{a}$, 


$$
\left\{\begin{array}{l}
B_{r}(r, \theta, z)=\frac{\mu_{0} I}{\pi} k^{2} a \sum_{n=1}^{\infty} n K_{n}^{\prime}(n k a) I_{n}^{\prime}(n k r) \sin [n(\theta-\varphi-k z)] \\
B_{\theta}(r, \theta, z)=\frac{\mu_{0} I}{\pi} k a \sum_{n=1}^{\infty} n K_{n}^{\prime}(n k a) \frac{I_{n}(n k r)}{r} \cos [n(\theta-\varphi-k z)] \\
B_{z}(r, \theta, z)=\frac{\mu_{0} I}{2 \pi} k-\frac{\mu_{0} I}{\pi} k^{2} a \sum_{n=1}^{\infty} n K_{n}^{\prime}(n k a) I_{n}(n k r) \cos [n(\theta-\varphi-k z)]
\end{array}\right.
$$

Similarly, for $r>a$,

$$
\left\{\begin{array}{l}
B_{r}(r, \theta, z)=\frac{\mu_{0} I}{\pi} k^{2} a \sum_{n=1}^{\infty} n I_{n}^{\prime}(n k a) K_{n}^{\prime}(n k r) \sin [n(\theta-\varphi-k z)] \\
B_{\theta}(r, \theta, z)=\frac{\mu_{0} I}{2 \pi r}+\frac{\mu_{0} I}{\pi} k a \sum_{n=1}^{\infty} n I_{n}^{\prime}(n k a) \frac{K_{n}(n k r)}{r} \cos [n(\theta-\varphi-k z)] \\
B_{z}(r, \theta, z)=-\frac{\mu_{0} I}{\pi} k^{2} a \sum_{n=1}^{\infty} n I_{n}^{\prime}(n k a) K_{n}(n k r) \cos [n(\theta-\varphi-k z)]
\end{array}\right.
$$

These results of magnetic fields are identical to those described in the reference $[7,8]$. Instead of the above-mentioned divergence-free vector potential, alternative forms of vector potential that individually are divergence-free in their respective regions $(r<a$ and $\mathrm{r}>\mathrm{a}$ ) [7], are not continuous across the boundary at $\mathrm{r}=\mathrm{a}$. However, such alternative forms can not be applied for the evaluation of the magnetic energy and the magnetic flux enclosed by the helical conductor.

\section{CONCLUSION}

The vector potential due to a single helical current carrying thin conductor can be directly derived from the definitive integral form, following the Caspi's mathematical treatment for the multipole field due to the helical wiggler magnet. The resultant expressions of the vector potential of divergence-free are useful for the evaluation of the magnetic energy and the magnetic flux enclosed by the helical conductor. As a result, it is realized that the fields produced by a helical current can be computed by first determining the vector potential, using the explicit integral expression, and then obtaining the magnetic field by taking the curl. 


\section{References}

[1] S. Caspi, "The Vector Potential and Stored Energy of Thin Cosine(n $\theta)$ Helical Wiggler", SC-MAG-529, LBL-38075, (1995).

[2] W. R. Smythe, "Static and Dynamic Electricity", McGraw-Hill, p.65 (1968).

[3] W. K. H. Panofsky and M. Phillips, "Classical Electricity and Magnetism", Addison-Wesley, p.75 (1962).

[4] I. S. Gradshteyn and I. M. Ryzhik, "Table of Integrals, Series, and Products", Academic Press, Eq. 3.754(2), p.419 (1980).

[5] G. N. Watson, "A Treatise on the Theory of Bessel Functions", Cambridge University Press, Sec. 11.3, Eq.(8), p.361, (1944).

[6] M. Abramowitz and I. A. Stegun (eds.), "Handbook of Mathematical Functions", Dover, pp.374-376 (1970).

[7] T. Tominaka, M. Okamura, and T. Katayama, "Analytical Field Calculation of Helical Coils", Nucl. Instr. \& Meth. A 459, pp.398-411 (2001).

[8] T. Tominaka, M. Okamura, and T. Katayama, "Analytical Field Calculation of Helical Magnets with an Axially Symmetric Yoke”, Nucl. Instr. \& Meth. A 484, pp.36-444 (2002). 
Figure captions

Fig. 1. Schematic view of a single helical current conductor.

Fig. 2. Cross section of a single helical current conductor at $\mathrm{z}=0$. 


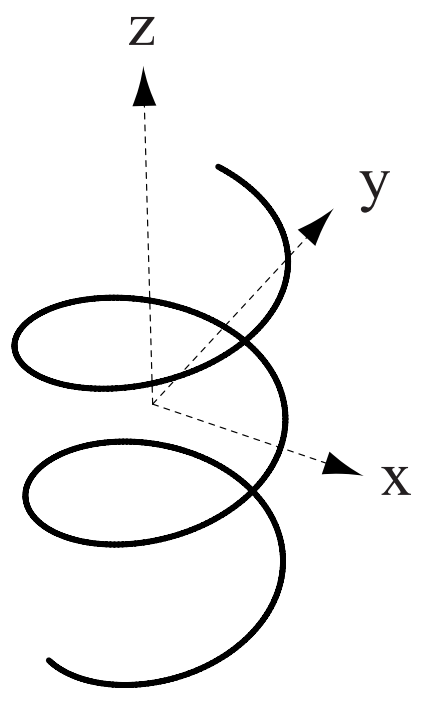

Fig. 1

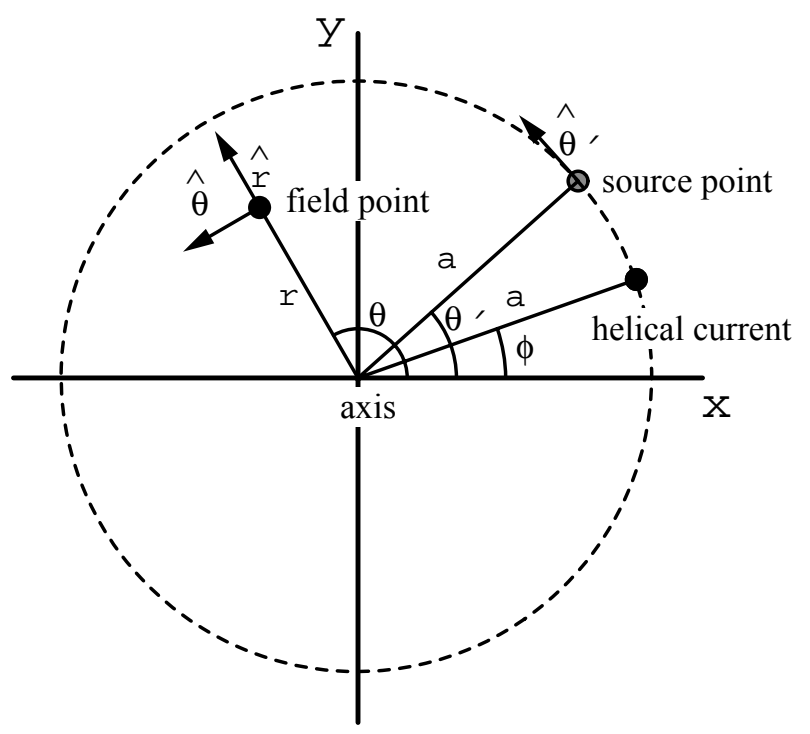

Fig. 2 